\title{
Thermodynamic and kinetic constraints on microbial mediated redox biogeochemistry
}

\author{
JIANQIU ZHENG ${ }^{1}$, TIM SCHEIBE ${ }^{2}$, VANESSA BAILEY ${ }^{3}$ \\ Pacific Northwest National Laboratory \\ 1jianqiu.zheng@pnnl.gov \\ 2tim.scheibe@pnnl.gov \\ ${ }^{3}$ Vanessa.Bailey@pnnl.gov
}

\begin{abstract}
The terrestrial-aquatic interfaces represent dynamic transition zones with sharp geochemical gradients across highly compressed temporal and spatial scales. Such unique habitats harbor large and diverse microbial populations that are actively regenerating redox gradients to sustain element cycling. The traditional view of stratified zonation of microbial mediated redox reactions, commonly referred to as the "redox ladder" is increasingly questioned, largely due to fine scale heterogeneity of physicochemical properties creating distinct energetic constraints on microbial community and their metabolic processes. We elucidate how the interplay between microbial communities and their surrounding environmental niche "organize" soil redox reactions by experimentally forcing static and dynamic redox conditions in microcosms. Co-occurrence of denitrification, iron reduction, sulfate reduction and methanogenesis were observed to various extents in experiments under suboxic, anoxic and dynamic-redox conditions, suggesting factors other than thermodynamic favorability are playing critical roles in regulating the organization of redox reactions. Numerical experiments using an aqueous explicit biogeochemical model demonstrate reaction kinetics play a key role in the determination of the sequence and cooccurrence of microbial redox reactions. Such kinetic modifications are likely manifested from microbe-specific physiological limitations, $\mathrm{pH}$ feedback, or community interactions. We conclude that ecological and physiological factors determining microbial reaction kinetics may be as important as thermodynamic assessments in predicting microbial response in dynamic redox environment.
\end{abstract}

